\title{
A framework for analysis of observer-based ILC
}

Johanna Wallén, Mikael Norrlöf, Svante Gunnarsson

Division of Automatic Control

E-mail: johanna@isy.liu.se, mino@isy.liu.se, svante@isy.liu.se

16th October 2009

Report no.: LiTH-ISY-R-2918

Accepted for publication in Symposium on Learning Control at CDC 2009, Shanghai, China

Address:

Department of Electrical Engineering

Linköpings universitet

SE-581 83 Linköping, Sweden

WWW: http://wwW.control.isy.liu.se

AUTOMATIC CONTROL

REGLERTEKNIK

LINKÖPINGS UNIVERSITET

Technical reports from the Automatic Control group in Linköping are available from http://www. control.isy.liu.se/publications. 


\begin{abstract}
A framework for Iterative Learning Control (ILC) is proposed for the situation when the ILC algorithm is based on an estimate of the controlled variable obtained from an observer-based estimation procedure. Under the assumption that the ILC input converges to a bounded signal, a general expression for the asymptotic error of the controlled variable is given. The asymptotic error is then exemplified by an ILC algorithm applied to a flexible two-mass model of a robot joint.
\end{abstract}

Keywords: Iterative learning control; Framework, Estimate, Asymptotic, Controlled variable 


\title{
A framework for analysis of observer-based ILC
}

\author{
Johanna Wallén, Mikael Norrlöf and Svante Gunnarsson
}

\begin{abstract}
A framework for Iterative Learning Control (ILC) is proposed for the situation when the ILC algorithm is based on an estimate of the controlled variable obtained from an observer-based estimation procedure. Under the assumption that the ILC input converges to a bounded signal, a general expression for the asymptotic error of the controlled variable is given. The asymptotic error is then exemplified by an ILC algorithm applied to a flexible two-mass model of a robot joint.
\end{abstract}

\section{INTRODUCTION}

The idea in Iterative Learning Control (ILC) is to compensate for errors when the system performs a repetitive motion. The first publications [1], [2], [3] regarding the control method were published in 1984, and in [4] a summary of the field and recent publications can be found. Traditionally ILC has been applied to systems where the controlled variable also is the measured variable. In industrial robot applications, for example plasma cutting in Fig. 1, this is typically not the case. In a standard industrial robot the angles seen from the motor side of the joints are measured. However, the control objective is to follow a desired tool path. Obviously it is a problem that the controlled variables cannot be measured, which is confirmed from ILC experiments performed on a robot, see for example [5]. This motivates the need of using estimates of the controlled variable in the ILC algorithm to be able to improve the performance.

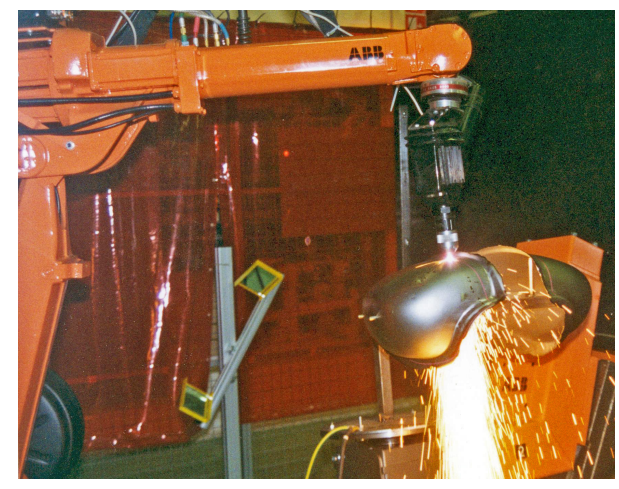

Fig. 1. A commercial industrial ABB robot IRB1400 performing plasma cutting [6]. Tool position and orientation cannot be measured due to practical and economical reasons. The control objective is however to follow a desired tool path, whereas the measured variables are the angles at the motor side of the joints.

This work was supported by ABB AB - Robotics, Vinnova's Industry Excellence Center LINK-SIC at Linköping University, and the Swedish Research Council (VR), which are gratefully acknowledged.

All authors are with the Division of Automatic Control, Department of Electrical Engineering, Linköping University, SE-58183 Linköping, Sweden, $\{j o h a n n a$, mino, svante\}@isy.liu.se
The aim of this paper is present a framework for the situation when an ILC algorithm is combined with a procedure for generating an estimate of the controlled variable. To the best of the authors knowledge, estimation techniques and ILC have only been combined in a few publications. One example is [7], where the ILC algorithm uses an estimate of the arm angle, computed using measurements of the motor angle and the arm angular acceleration of a flexible one-link robot arm. The study [8] also discusses these issues, based on simulations of a realistic robot model. In [9] the model error of a state-space model linearised along the desired trajectory is estimated using a Kalman filter in the iterationdomain. The control signal at next iteration is then given by minimising the deviation of the states from the desired trajectory. Another example is [10], where the estimated states for a class of time-varying nonlinear systems are used in an ILC algorithm and the asymptotic behaviour of the system is discussed. The focus in these papers are on specific estimation and/or ILC algorithm techniques, while the following aspects are of main interest in this paper:

- A framework for analysis of the properties of the ILC algorithm when an estimate of the controlled variable is used in the ILC algorithm.

- An expression for asymptotic error of the controlled variable when the ILC algorithm based on an estimate of the controlled variable has converged.

The paper is organised as follows. Section II presents the system description, and Section III contains a description of the procedure for estimating the controlled variable. In Section IV the considered class of ILC algorithms is presented, and Section $\mathrm{V}$ presents the key results concerning the properties of the ILC algorithm. The obtained results are illustrated in Section VI, and finally Section VII presents some conclusions.

\section{SYSTEM DESCRIPTION}

\section{A. Notation and prerequisites}

All signals are defined on a finite time interval $t=n t_{s}$, $n \in\{0, \ldots, N-1\}$ with $N$ number of samples and sampling interval $t_{s}$. The forward time-shift operator $q$ is defined as $q u(t)=u\left(t+t_{s}\right)$. Throughout the paper equations are in the time-domain and the arguments $t$ and $q$ are often omitted for convenience in the text when there is no risk of confusion.

\section{B. System description}

Consider the system shown in Fig. 2. The linear, timeand iteration-invariant discrete-time system $T$ has two inputs; a reference signal $r$ and an ILC input signal $u_{k}$, with $k$ denoting the iteration number. The outputs are the measured 


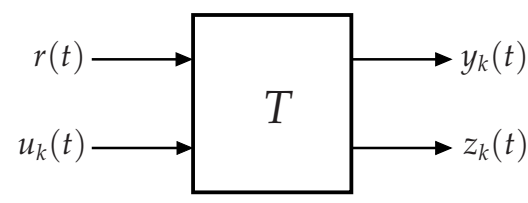

Fig. 2. Description of the system $T$ with reference $r(t)$, ILC input $u_{k}(t)$, measured variable $y_{k}(t)$ and controlled variable $z_{k}(t)$ at iteration $k$.

variable $y_{k}$ and the controlled variable $z_{k}$. The system $T$ can have internal feedback, which means that $T$ contains the system to be controlled as well as the controller. Denoting the true system by the superscript " 0 ", the system description at iteration $k$ is

$$
\begin{aligned}
& y_{k}(t)=T_{r y}^{0}(q) r(t)+T_{u y}^{0}(q) u_{k}(t) \\
& z_{k}(t)=T_{r z}^{0}(q) r(t)+T_{u z}^{0}(q) u_{k}(t)
\end{aligned}
$$

The measured variable $y_{k}$ is given from (1), whereas the controlled variable $z_{k}$ is described by the relation (2). The systems $T_{r y}^{0}, T_{u y}^{0}, T_{r z}^{0}$ and $T_{u z}^{0}$ are assumed to be stable and causal. System and measurement disturbances are not included here for simplicity reasons.

Example 1 (Open-loop system). First, an example of the description (1) - (2) is given by the stable open-loop system shown in Fig. 3. The true system, denoted $G^{0}$, is described by the discrete-time state-space system

$$
\begin{aligned}
& x\left(t+t_{s}\right)=A^{0} x(t)+B^{0} u_{k}(t) \\
& y_{k}(t)=C^{0} x(t) \\
& z_{k}(t)=M^{0} x(t)
\end{aligned}
$$

with the ILC input signal $u_{k}$ at iteration $k$ as input. The outputs are given by the measured variable $y_{k}$ and controlled variable $z_{k}$ at iteration $k$. From (3) the transfer operators $T_{u y}^{0}$ and $T_{u z}^{0}$ are given by

$$
\begin{aligned}
& T_{u y}^{0}=C^{0}\left(q I-A^{0}\right)^{-1} B^{0} \\
& T_{u z}^{0}=M^{0}\left(q I-A^{0}\right)^{-1} B^{0}
\end{aligned}
$$

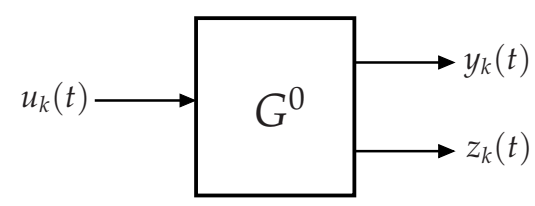

Fig. 3. Stable open-loop system; an example of the system description (1) - (2) with input $u_{k}(t)$ and outputs $y_{k}(t), z_{k}(t)$ at iteration $k$.

Example 2 (Closed-loop system). The system description (1) - (2) with inputs $r, u_{k}$ and outputs $y_{k}, z_{k}$ can also be exemplified by the closed-loop system shown in Fig. 4. It means that the system $T$ has internal feedback and contains both the system to be controlled and the controller in operation. In a real application the controller structure can be more complex than the one shown in Fig. 4, and the structure considered here is used for illustration.
The system to be controlled is given by the state-space description (3), however now with the input $\bar{u}_{k}$ which is the control signal generated by the controller $F$ at iteration $k$. The transfer operators (4) are in a similar manner denoted $T_{\bar{u} y}^{0}$ and $T_{\bar{u}}^{0}$. Throughout the paper it is assumed that the controller $F$ is given, and that the closed-loop system is stable. Noting the choice of adding the ILC input $u_{k}$ to the reference $r$ in this example, it gives the closed-loop system from $r, u_{k}$ to $y_{k}$ and $z_{k}$ as

$$
\begin{aligned}
y_{k}(t) & =\frac{F(q) T_{\bar{u} y}^{0}(q)}{1+F(q) T_{\bar{u} y}^{0}(q)} r(t)+\frac{F(q) T_{\bar{u} y}^{0}(q)}{1+F(q) T_{\bar{u} y}^{0}(q)} u_{k}(t) \\
& =T_{r y}^{0}(q) r(t)+T_{u y}^{0}(q) u_{k}(t) \\
z_{k}(t) & =\frac{F(q) T_{\bar{u} z}^{0}(q)}{1+F(q) T_{\bar{u} y}^{0}(q)} r(t)+\frac{F(q) T_{\bar{u} z}^{0}(q)}{1+F(q) T_{\bar{u} y}^{0}(q)} u_{k}(t) \\
& =T_{r z}^{0}(q) r(t)+T_{u z}^{0}(q) u_{k}(t)
\end{aligned}
$$

from which the transfer operators $T_{r y}^{0}, T_{u y}^{0}, T_{r z}^{0}$ and $T_{u z}^{0}$ are identified from (1) - (2). They are stable according to the assumption about the closed-loop system.

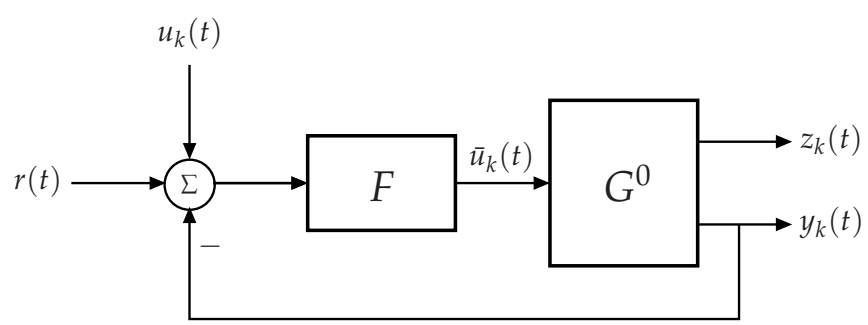

Fig. 4. Closed-loop system; an example of the system description (1) - (2) with inputs $r(t), u_{k}(t)$ and outputs $y_{k}(t), z_{k}(t)$ at iteration $k$. The system $G^{0}$ is controlled by $\bar{u}_{k}(t)$ from the controller $F$.

Finally it can be noted that the system description (1) - (2) is a natural extension of the description (system and measurement disturbances omitted here) presented in [11], where

$$
y_{k}(t)=T_{r}(q) r(t)+T_{u}(q) u_{k}(t)
$$

In [11] the properties of this system, when controlled by an ILC algorithm, are analysed with focus on the measured variable $y_{k}$ used in the ILC algorithm.

\section{System description in matrix form}

For the analysis in Section $\mathrm{V}$, the matrix description of the system and the ILC algorithm is used, which is closely related to the descriptions of systems using ILC in [12], [13] and [14] among others. First, define the vector $\boldsymbol{r}$ of the $N$ sample sequence of the reference signal $r$ as

$$
\boldsymbol{r}=\left(\begin{array}{lll}
r(0) & \ldots & r\left((N-1) t_{s}\right)
\end{array}\right)^{T}
$$

which for simplicity will be denoted

$$
\boldsymbol{r}=\left(\begin{array}{lll}
r_{0} & \ldots & r_{N-1}
\end{array}\right)^{T}
$$

Next, define the vectors $\boldsymbol{u}_{k}, \boldsymbol{y}_{k}$ and $\boldsymbol{z}_{k}$ similarly. The matrix $\boldsymbol{T}_{r y}^{0}$ is formed by the pulse response coefficients $g_{T_{r y}^{0}}$, $t \in\left\{0, \ldots,(N-1) t_{s}\right\}$ of the transfer operator $T_{r y}^{0}$ in $(1)$, 
denoted $g_{T_{r y}^{0}, 0}, \ldots, g_{T_{r y}^{0}, N-1}$. This results in the $N \times N$ Toeplitz matrix

$$
\boldsymbol{T}_{r y}^{0}=\left(\begin{array}{cccc}
g_{T_{r y}, 0} & 0 & \cdots & 0 \\
g_{T_{r y}, 1} & g_{T_{r y}, 0} & & 0 \\
\vdots & & \ddots & \vdots \\
g_{T_{r y}, N-1} & g_{T_{r y}, N-2} & \cdots & g_{T_{r y}, 0}
\end{array}\right)
$$

and the other system matrices $\boldsymbol{T}_{u y}^{0}, \boldsymbol{T}_{r z}^{0}$ and $\boldsymbol{T}_{u z}^{0}$ are defined similarly. It can be noted that they are lowertriangular matrices, since the transfer operators $T_{r y}^{0}, T_{u y}^{0}, T_{r z}^{0}$ and $T_{u z}^{0}$ in (1) - (2) are causal. Finally, the system description (1) - (2) of the measured variable $y_{k}$ and controlled variable $z_{k}$ is rewritten in matrix form as

$$
\begin{aligned}
& \boldsymbol{y}_{k}=\boldsymbol{T}_{r y}^{0} \boldsymbol{r}+\boldsymbol{T}_{u y}^{0} \boldsymbol{u}_{k} \\
& \boldsymbol{z}_{k}=\boldsymbol{T}_{r z}^{0} \boldsymbol{r}+\boldsymbol{T}_{u z}^{0} \boldsymbol{u}_{k}
\end{aligned}
$$

This formulation is more general than the LTI representation (1) - (2) because it covers both time-invariant as well as time-varying systems. However, for time-varying systems the matrices $\boldsymbol{T}_{r y}^{0}, \boldsymbol{T}_{u y}^{0}, \boldsymbol{T}_{r z}^{0}$ and $\boldsymbol{T}_{u z}^{0}$ are not lower-triangular Toeplitz matrices, but instead general lower-triangular matrices. The vectors and matrices describing the system in matrix form are throughout the paper written in bold face, to easily distinguish the matrix description of the system from the description using transfer operators.

\section{ESTIMATION OF THE CONTROLLED VARIABLE}

In the system description (1) - (2) the measured variable $y_{k}$ and controlled variable $z_{k}$ are involved. Obviously, if applying an ILC input $u_{k}$ on the system, based directly on the measured variable, it will drive the controlled variable towards an incorrect value since the dynamics between $y_{k}$ and $z_{k}$ is neglected. It is therefore natural to use an estimate of $z_{k}$, denoted $\hat{z}_{k}$, in the ILC algorithm. We propose the following parameterisation where the estimate $\hat{z}_{k}$ is related to the reference $r$, ILC input $u_{k}$ and measured variable $y_{k}$ through

$$
\hat{z}_{k}(t)=F_{r}(q) r(t)+F_{u}(q) u_{k}(t)+F_{y}(q) y_{k}(t)
$$

where the filters $F_{r}, F_{u}$ and $F_{y}$ are assumed to be stable. See Fig. 5 for an illustration of the system $T$ together with the estimation procedure (10).

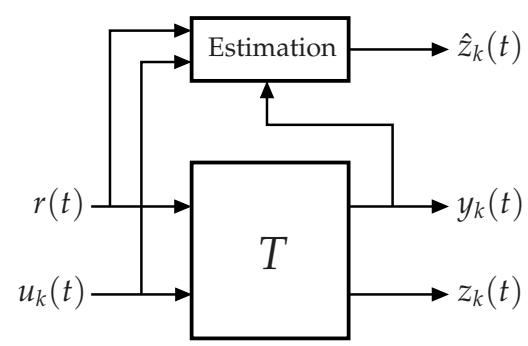

Fig. 5. Description of the system $T$ with reference $r(t)$, ILC input $u_{k}(t)$, measured variable $y_{k}(t)$ and controlled variable $z_{k}(t)$ at iteration $k$. The estimation procedure (10) results in an estimate $\hat{z}_{k}$ of the controlled variable.
The relation (10) will now be illustrated by some examples, showing how the estimate $\hat{z}_{k}$ can be created. The cases are also discussed in more detail in Section VI.

Example 3 (Case A - Measured variable). A naive way of estimating $z_{k}$ would be to neglect the dynamics between the measured variable $y_{k}$ and controlled variable $z_{k}$ and to put

$$
\hat{z}_{k}(t)=y_{k}(t)
$$

which means choosing

$$
F_{r}(q)=0, \quad F_{u}(q)=0, \quad F_{y}(q)=1
$$

in (10).

Example 4 (Case B - Estimate from model of direct relation between measured and controlled variable). There are situations where the relationship between the measured variable $y_{k}$ and controlled variable $z_{k}$ can be explicitly described by the discrete-time relation

$$
z_{k}(t)=T_{y z}^{0}(q) y_{k}(t)
$$

(See Appendix for an example where (11) holds.) By using a nominal model of the relationship (11) between $y_{k}$ and $z_{k}$, it implies the estimate

$$
\hat{z}_{k}(t)=T_{y z}(q) y_{k}(t)
$$

which can be incorporated in the description (10) by choosing

$$
F_{r}(q)=0, \quad F_{u}(q)=0, \quad F_{y}(q)=T_{y z}(q)
$$

In the last example an observer is used to estimate $z_{k}$. The idea with an observer is that information from additional sensors can be fused with the original measurements available, see for example [15]. One example where estimation techniques is used together with ILC is [8]. The arm angle of a realistic robot model is estimated by an extended Kalman filter (EKF) based on measurements of motor angle and acceleration of the tool. The estimate is thereafter used in an ILC algorithm.

Example 5 (Case C - Estimate from observer). Based on a nominal model of the state space description (3) an observer can be formed as

$$
\begin{aligned}
\hat{x}\left(t+t_{s}\right) & =A \hat{x}(t)+B \bar{u}_{k}(t)+K\left(y_{k}(t)-C \hat{x}(t)\right) \\
\hat{z}_{k}(t) & =M \hat{x}(t)
\end{aligned}
$$

Using transfer operators the estimate of the controlled variable can be expressed

$$
\begin{aligned}
\hat{z}_{k}(t)= & M(q I-(A-K C))^{-1} B \bar{u}_{k}(t) \\
& +M(q I-(A-K C))^{-1} K y_{k}(t) \\
= & F_{\bar{u}}(q) \bar{u}_{k}(t)+F_{\bar{y}}(q) y_{k}(t)
\end{aligned}
$$


From the closed-loop system in Fig. 4 it can then be seen that the filters in (10) can be identified as

$$
\begin{aligned}
\hat{z}_{k}(t)= & F_{\bar{u}}(q)\left(F(q) u_{k}(t)+F(q) r(t)-F(q) y_{k}(t)\right) \\
& \quad+F_{y}(q) y_{k}(t) \\
= & F_{\bar{u}}(q) F(q) r(t)+F_{\bar{u}}(q) F(q) u_{k}(t) \\
& \quad+\left(F_{\bar{y}}(q)-F_{\bar{u}}(q) F(q)\right) y_{k}(t) \\
= & F_{r}(q) r(t)+F_{u}(q) u_{k}(t)+F_{y}(q) y_{k}(t)
\end{aligned}
$$

To summarise, from Examples 3 - 5 above it can be seen that the description (10) incorporates different ways of generating the estimate $\hat{z}_{k}$ by choosing the filters $F_{r}, F_{u}$ and $F_{y}$ in different ways; by neglecting the dynamics, using a nominal model of the relation between $y_{k}$ and $z_{k}$, and from observers. Therefore it is natural to use the relation (10) in the ILC algorithm, as is seen in the forthcoming section.

Now, define the vectors of the N-sample sequence of the estimate $\hat{z}_{k}$ similarly as in (6), and form the matrices $\boldsymbol{F}_{r}, \boldsymbol{F}_{u}$ and $\boldsymbol{F}_{y}$ similarly as in (7) by the pulse response coefficients of the filters $F_{r}, F_{u}$ and $F_{y}$. It results in the matrix description of the relation (10) as follows

$$
\hat{\boldsymbol{z}}_{k}=\boldsymbol{F}_{r} \boldsymbol{r}+\boldsymbol{F}_{u} \boldsymbol{u}_{k}+\boldsymbol{F}_{y} \boldsymbol{y}_{k}
$$

\section{ILC ALGORITHM}

The update equation for an ILC algorithm is in transferoperator form given by

$$
u_{k+1}(t)=Q(q)\left(u_{k}(t)+L(q) \epsilon_{k}(t)\right)
$$

where the linear filters $Q$ and $L$ are possibly non-causal. The error used in the ILC algorithm,

$$
\epsilon_{k}(t)=r(t)-\hat{z}_{k}(t)
$$

is the difference between the reference signal $r$ and the estimate $\hat{z}_{k}$ in (10) of the controlled variable at iteration $k$. In conjunction with the error $\epsilon_{k}$ in (17) used in the ILC algorithm, the error of the controlled variable,

$$
e_{k}(t)=r(t)-z_{k}(t)
$$

is used to evaluate the performance of the system.

Rewrite in matrix form by first defining the vectors $\boldsymbol{u}_{k+1}, \boldsymbol{\epsilon}_{k}$ and $\boldsymbol{e}_{k}$ similarly as in (6). Using the operators $\boldsymbol{L}$ and $Q$, it results in the ILC update equation and error used in the ILC algorithm

$$
\begin{aligned}
\boldsymbol{u}_{k+1} & =\boldsymbol{Q}\left(\boldsymbol{u}_{k}+\boldsymbol{L} \boldsymbol{\epsilon}_{k}\right) \\
\boldsymbol{\epsilon}_{k} & =\boldsymbol{r}-\hat{\boldsymbol{z}}_{k}
\end{aligned}
$$

while the system performance (18) is given by

$$
\boldsymbol{e}_{k}=\boldsymbol{r}-\boldsymbol{z}_{k}
$$

Note that the matrices $\boldsymbol{L}$ and $\boldsymbol{Q}$ are not necessarily lower triangular, since the ILC algorithm can be non-causal.

\section{ANALYSIS}

To support the analysis and make it easier to follow the derivations in this section, the main equations (8) - (9), (15), and (19) - (21), from the previous sections are summarised here. The system given by

$$
\begin{aligned}
& \boldsymbol{y}_{k}=\boldsymbol{T}_{r y}^{0} \boldsymbol{r}+\boldsymbol{T}_{u y}^{0} \boldsymbol{u}_{k} \\
& \boldsymbol{z}_{k}=\boldsymbol{T}_{r z}^{0} \boldsymbol{r}+\boldsymbol{T}_{u z}^{0} \boldsymbol{u}_{k}
\end{aligned}
$$

is controlled using the ILC input signal $\boldsymbol{u}_{k}$, updated by

$$
\begin{aligned}
\boldsymbol{u}_{k+1} & =\boldsymbol{Q}\left(\boldsymbol{u}_{k}+\boldsymbol{L} \boldsymbol{\epsilon}_{k}\right) \\
\boldsymbol{\epsilon}_{k} & =\boldsymbol{r}-\hat{\boldsymbol{z}}_{k}
\end{aligned}
$$

using the estimate

$$
\hat{\boldsymbol{z}}_{k}=\boldsymbol{F}_{r} \boldsymbol{r}+\boldsymbol{F}_{u} \boldsymbol{u}_{k}+\boldsymbol{F}_{y} \boldsymbol{y}_{k}
$$

From the relations (22) - (26) above it is now possible to derive the ILC system equation which will be used in the stability analysis.

Lemma 1 (ILC system equation). Having a system as in (22) - (23) using the ILC update equation in (24) and the estimate of $z_{k}$ from (26), the ILC system equation is given by

$$
\boldsymbol{u}_{k+1}=\boldsymbol{H} \boldsymbol{u}_{k}+\boldsymbol{H}_{r} \boldsymbol{r}
$$

where

$$
\begin{aligned}
& \boldsymbol{H}=\boldsymbol{Q}\left(I-\boldsymbol{L}\left(\boldsymbol{F}_{u}+\boldsymbol{F}_{y} \boldsymbol{T}_{u y}^{0}\right)\right) \\
& \boldsymbol{H}_{r}=\boldsymbol{Q} \boldsymbol{L}\left(I-\left(\boldsymbol{F}_{r}+\boldsymbol{F}_{y} \boldsymbol{T}_{r y}^{0}\right)\right)
\end{aligned}
$$

Proof. Using (22) and (26), the error $\epsilon_{k}$ in (25) can be written

$$
\begin{aligned}
\boldsymbol{\epsilon}_{k} & =\boldsymbol{r}-\boldsymbol{F}_{r} \boldsymbol{r}-\boldsymbol{F}_{u} \boldsymbol{u}_{k}-\boldsymbol{F}_{y}\left(\boldsymbol{T}_{r y}^{0} \boldsymbol{r}+\boldsymbol{T}_{u y}^{0} \boldsymbol{u}_{k}\right) \\
& =\left(I-\left(\boldsymbol{F}_{r}+\boldsymbol{F}_{y} \boldsymbol{T}_{r y}^{0}\right)\right) \boldsymbol{r}-\left(\boldsymbol{F}_{u}+\boldsymbol{F}_{y} \boldsymbol{T}_{u y}^{0}\right) \boldsymbol{u}_{k}
\end{aligned}
$$

Inserting the error $\epsilon_{k}$ into the ILC update equation (24) then gives

$$
\begin{aligned}
\boldsymbol{u}_{k+1}= & \boldsymbol{Q}\left(I-\boldsymbol{L}\left(\boldsymbol{F}_{u}+\boldsymbol{F}_{y} \boldsymbol{T}_{u y}^{0}\right)\right) \boldsymbol{u}_{k} \\
& +\boldsymbol{Q} \boldsymbol{L}\left(I-\left(\boldsymbol{F}_{r}+\boldsymbol{F}_{y} \boldsymbol{T}_{r y}^{0}\right)\right) \boldsymbol{r}
\end{aligned}
$$

and the result follows.

Stability of the ILC system in Lemma 1 can be expressed based on the results in [11].

Theorem 2 (Stability, iteration-invariant system). Consider the ILC system equation from Lemma 1,

$$
\boldsymbol{u}_{k+1}=\boldsymbol{H} \boldsymbol{u}_{k}+\boldsymbol{H}_{r} \boldsymbol{r}
$$

This system is stable if and only if $\rho(\boldsymbol{H})<1$, where $\rho(\cdot)$ is the spectral radius of the matrix.

Proof. See [11]; Theorem 1 and proof of Corollary 3.

Given a reference trajectory $r(t)$ on a finite time interval $t \in\left[0, t_{f}\right]$ with a piecewise continuous derivative, there exists a unique input trajectory $u_{d}$ on the same time interval 
such that $z$ equals $r$. Stability in the sense of Theorem 2 means that it exists a $\delta<\infty$, see [11], such that

$$
\sup _{k=0,1, \ldots}\left\|\boldsymbol{u}_{d}-\boldsymbol{u}_{k}\right\|<\delta
$$

Lemma 3 (Asymptotic behaviour). Under the assumption that the input $\boldsymbol{u}_{k}$ in the ILC system equation from Lemma 1 converges to a bounded signal as $k \rightarrow \infty$, the limit is given by

$$
\begin{aligned}
\boldsymbol{u}_{\infty}=\left(I-\boldsymbol{Q}\left(I-\boldsymbol{L}\left(\boldsymbol{F}_{u}+\boldsymbol{F}_{y} \boldsymbol{T}_{u y}^{0}\right)\right)\right)^{-1} \\
\cdot \boldsymbol{Q} \boldsymbol{L}\left(I-\left(\boldsymbol{F}_{r}+\boldsymbol{F}_{y} \boldsymbol{T}_{r y}^{0}\right)\right) \boldsymbol{r}
\end{aligned}
$$

The corresponding asymptotic error $\boldsymbol{r}-\boldsymbol{z}_{\infty}$ becomes

$$
\begin{array}{r}
\boldsymbol{e}_{\infty}=\left(I-\boldsymbol{T}_{r z}^{0}-\boldsymbol{T}_{u z}^{0}\left(I-\boldsymbol{Q}\left(I-\boldsymbol{L}\left(\boldsymbol{F}_{u}+\boldsymbol{F}_{y} \boldsymbol{T}_{u y}^{0}\right)\right)\right)^{-1}\right. \\
\left.\cdot \boldsymbol{Q} \boldsymbol{L}\left(I-\left(\boldsymbol{F}_{r}+\boldsymbol{F}_{y} \boldsymbol{T}_{r y}^{0}\right)\right)\right) \boldsymbol{r}
\end{array}
$$

Proof. Let $k \rightarrow \infty$ and solve the ILC system equation from Lemma 1,

$$
\begin{aligned}
\boldsymbol{u}_{\infty}= & \boldsymbol{Q}\left(I-\boldsymbol{L}\left(\boldsymbol{F}_{u}+\boldsymbol{F}_{y} \boldsymbol{T}_{u y}^{0}\right)\right) \boldsymbol{u}_{\infty} \\
& +\boldsymbol{Q} \boldsymbol{L}\left(I-\left(\boldsymbol{F}_{r}+\boldsymbol{F}_{y} \boldsymbol{T}_{r y}^{0}\right)\right) \boldsymbol{r}
\end{aligned}
$$

for $\boldsymbol{u}_{\infty}$. Insert $\boldsymbol{u}_{\infty}$ in the relation for the error of the controlled variable, using (23),

$$
e_{\infty}=r-z_{\infty}=r-\left(\boldsymbol{T}_{r z}^{0} r+\boldsymbol{T}_{u z}^{0} \boldsymbol{u}_{\infty}\right)
$$

to derive the asymptotic error.

From a design perspective the following result has an important impact.

Theorem 4 (Monotone convergence). Consider the same ILC system as in Theorem 2. If the maximum singular value fulfils $\bar{\sigma}(\boldsymbol{H}) \leq \lambda<1$, then the ILC system is stable, and

$$
\left\|\boldsymbol{u}_{\infty}-\boldsymbol{u}_{k}\right\| \leq \lambda^{k}\left\|\boldsymbol{u}_{\infty}-\boldsymbol{u}_{0}\right\|
$$

with $\boldsymbol{u}_{\infty}$ from (27) in Lemma 3.

Proof. See proof of Theorem 9 in [11] and Lemma 3 above for the computation of $\boldsymbol{u}_{\infty}$.

Monotone convergence of $\boldsymbol{u}_{k}$ is a good design criterion, but it is also necessary to check $e_{0}$ and $e_{\infty}$ so that the error is reduced as desired. From Theorem 4 it can be seen that the smaller the maximum singular value of the matrix $\boldsymbol{H}$, the faster the convergence. A design criterion therefore should include minimising the maximum singular value $\bar{\sigma}(\boldsymbol{H})$ as well as the asymptotic error $\boldsymbol{e}_{\infty}$.

\section{ILLUSTRATION OF THE RESULTS}

The system description (22) - (26) derived in the paper, and the asymptotic error $\boldsymbol{e}_{\infty}$ from Lemma 3 are discussed in this section. To support the intuition of the results, the relations are illustrated for a system where it is possible to write an explicit discrete-time relationship between the measured variable $y_{k}$ and controlled variable $z_{k}$ as in (11),

$$
z_{k}(t)=T_{y z}^{0}(q) y_{k}(t)
$$

See Appendix for further details, where such a system is exemplified by a flexible two-mass model of a single robot joint. From the pulse response coefficients of the system $T_{y z}^{0}$, the matrix $\boldsymbol{T}_{y z}^{0}$ can be derived similarly as in (7), giving

$$
\boldsymbol{z}_{k}=\boldsymbol{T}_{y z}^{0} \boldsymbol{y}_{k}
$$

The ILC algorithm (24) is in this section applied to the system (22) - (23) for the following cases:

A. The ILC algorithm is based on $\boldsymbol{\epsilon}_{k}=\boldsymbol{r}-\boldsymbol{y}_{k}$, that is, the dynamics between $\boldsymbol{y}_{k}$ and $\boldsymbol{z}_{k}$ is neglected.

B. The ILC algorithm uses $\boldsymbol{\epsilon}_{k}=\boldsymbol{r}-\boldsymbol{T}_{y z} \boldsymbol{y}_{k}$, that is, $\hat{\boldsymbol{z}}_{k}$ is estimated from a model of the relation (29).

C. The ILC signal is updated using $\boldsymbol{\epsilon}_{k}=\boldsymbol{r}-\hat{\boldsymbol{z}}_{k}$ where $\hat{\boldsymbol{z}}_{k}=\boldsymbol{F}_{r} \boldsymbol{r}+\boldsymbol{F}_{u} \boldsymbol{u}_{k}+\boldsymbol{F}_{y} \boldsymbol{y}_{k}$ and the filters are obtained from the observer (12).

D. As a comparison, in order to show what ideally can be achieved, it is assumed that the controlled variable $\boldsymbol{z}_{k}$ can be measured and that the ILC algorithm is based on $\boldsymbol{\epsilon}_{k}=\boldsymbol{r}-\boldsymbol{z}_{k}$.

The choices of filters $F_{r}, F_{u}$ and $F_{y}$ in (10) to implement the cases $\mathrm{A}-\mathrm{C}$ were previously discussed in Examples $3-5$ given in Section III. In Table I the filters $\boldsymbol{H}$ and $\boldsymbol{H}_{r}$ in the ILC system equation, given in Lemma 1, together with the asymptotic error $e_{\infty}$ of the controlled variable $z_{k}$, from Lemma 3, are summarised for the cases above. These relations are discussed in more detail next.

\section{A. Case A - ILC using measured variable}

The first case to investigate is when the dynamics between the measured variable $\boldsymbol{y}_{k}$ and the controlled variable $\boldsymbol{z}_{k}$ is neglected, which gives the estimate $\hat{\boldsymbol{z}}_{k}=\boldsymbol{y}_{k}$, see Example 3. However, their actual relation is given by the equations $(22)-(23)$.

By using this estimate, it results in the error $\boldsymbol{\epsilon}_{k}=\boldsymbol{r}-\boldsymbol{y}_{k}$ in the ILC algorithm (24). Assume that $\boldsymbol{L}$ can be chosen such that $\boldsymbol{u}_{k}$ converges to a bounded signal when $\boldsymbol{Q}=I$ in the algorithm. This choice results in $\boldsymbol{\epsilon}_{\infty}=\mathbf{0}$ when the algorithm has converged. The asymptotic error $\boldsymbol{e}_{\infty}$ from Lemma 3 is then given by

$$
\boldsymbol{e}_{\infty}=\left(I-\boldsymbol{T}_{r z}^{0}-\boldsymbol{T}_{u z}^{0}\left(\boldsymbol{L} \boldsymbol{T}_{u y}\right)^{-1} \boldsymbol{L}\left(I-\boldsymbol{T}_{r y}^{0}\right)\right) \boldsymbol{r}
$$

Now assuming that the system can be described with an explicit relation between the measured and controlled variable as in (29). This results in the approximate relations for the finite-dimensional $(N \times N)$ matrices

$$
\boldsymbol{T}_{r z}^{0}=\boldsymbol{T}_{y z}^{0} \boldsymbol{T}_{r y}^{0}, \quad \boldsymbol{T}_{u z}^{0}=\boldsymbol{T}_{y z}^{0} \boldsymbol{T}_{u y}^{0}
$$

For the time-invariant SISO system described by (1) and (11), the relation (30) can using (31), and rewritten in transferoperator form, be simplified to

$$
e_{\infty}(t)=\left(1-T_{y z}^{0}(q)\right) r(t)
$$

Whenever $T_{y z}^{0} \neq 1$, this results in an asymptotic error $e_{\infty} \neq 0$ of the controlled variable, as expected.

There are better ways of estimating $\hat{\boldsymbol{z}}_{k}=\boldsymbol{y}_{k}$ than just neglecting the dynamics between $\boldsymbol{y}_{k}$ and $\boldsymbol{z}_{k}$. The two following sections discuss model-based estimates as described in Examples 4 and 5 in Section III. 


\begin{tabular}{|l||l|l|l|}
\hline & $\boldsymbol{\epsilon}_{k}$ & $\boldsymbol{u}_{k+1}=\boldsymbol{H} \boldsymbol{u}_{k}+\boldsymbol{H}_{r} \boldsymbol{r}$ & $e_{\infty}(t)=r(t)-z_{\infty}(t), \quad Q(q)=1$ \\
\hline \hline A & $\boldsymbol{r}-\boldsymbol{y}_{k}$ & $\begin{array}{l}\boldsymbol{H}=\boldsymbol{Q}\left(I-\boldsymbol{L} \boldsymbol{T}_{u y}^{0}\right) \\
\boldsymbol{H}_{r}=\boldsymbol{Q} \boldsymbol{L}\left(I-\boldsymbol{T}_{r y}^{0}\right)\end{array}$ & $\left(1-T_{y z}^{0}(q)\right) r(t)$ \\
\hline B & $\boldsymbol{r}-\boldsymbol{T}_{y z} \boldsymbol{y}_{k}$ & $\begin{array}{l}\boldsymbol{H}=\boldsymbol{Q}\left(I-\boldsymbol{L} \boldsymbol{T}_{y z} \boldsymbol{T}_{u y}^{0}\right) \\
\boldsymbol{H}_{r}=\boldsymbol{Q} \boldsymbol{L}\left(I-\boldsymbol{T}_{y z} \boldsymbol{T}_{r y}^{0}\right)\end{array}$ & $\left(1-T_{y z}^{0}(q) T_{y z}^{-1}(q)\right) r(t)$ \\
\hline C & $\boldsymbol{r}-\left(\boldsymbol{F}_{r} \boldsymbol{r}+\boldsymbol{F}_{u} \boldsymbol{u}_{k}+\boldsymbol{F}_{y} \boldsymbol{y}_{k}\right)$ & $\boldsymbol{H}=\boldsymbol{Q}\left(I-\boldsymbol{L}\left(\boldsymbol{F}_{u}+\boldsymbol{F}_{y} \boldsymbol{T}_{u y}^{0}\right)\right)$ & $\left(1-T_{y z}^{0}(q) \frac{T_{r y}^{0}(q) F_{u}(q)+\left(1-F_{r}(q)\right) T_{u y}^{0}(q)}{F_{u}(q)+F_{y}(q) T_{u y}^{0}(q)}\right) r(t)$ \\
& & $\boldsymbol{H}_{r}=\boldsymbol{Q} \boldsymbol{L}\left(I-\left(\boldsymbol{F}_{r}+\boldsymbol{F}_{y} \boldsymbol{T}_{r y}^{0}\right)\right)$ & \\
\hline D & $\boldsymbol{r}-\boldsymbol{z}_{k}$ & $\begin{array}{l}\boldsymbol{H}=\boldsymbol{Q}\left(I-\boldsymbol{L} \boldsymbol{T}_{y z}^{0} \boldsymbol{T}_{u y}^{0}\right) \\
\boldsymbol{H}_{r}=\boldsymbol{Q} \boldsymbol{L}\left(I-\boldsymbol{T}_{y z}^{0} \boldsymbol{T}_{r y}^{0}\right)\end{array}$ & 0 \\
\hline
\end{tabular}

B. Case B - ILC estimate from model of direct relation between $\boldsymbol{y}_{k}$ and $\boldsymbol{z}_{k}$

Under the assumption that the relation (29) between $\boldsymbol{y}_{k}$ and $\boldsymbol{z}_{k}$ holds, a straightforward way to estimate $\boldsymbol{z}_{k}$ is by using the nominal dynamics, which gives $\hat{z}_{k}=\boldsymbol{T}_{y z} \boldsymbol{y}_{k}$. The asymptotic error $\boldsymbol{e}_{\infty}$ from Lemma 3 , for $\boldsymbol{Q}=I$ and using the approximation (31), results in

$$
\begin{aligned}
\boldsymbol{e}_{\infty}=\left(I-\boldsymbol{T}_{y z}^{0}\left(\boldsymbol{T}_{r y}^{0}+\boldsymbol{T}_{u y}^{0}\left(\boldsymbol{L} \boldsymbol{T}_{y z} \boldsymbol{T}_{u y}^{0}\right)^{-1}\right.\right. \\
\left.\left.\cdot \boldsymbol{L}\left(I-\boldsymbol{T}_{y z} \boldsymbol{T}_{r y}^{0}\right)\right)\right) \boldsymbol{r}
\end{aligned}
$$

under the assumption that the ILC algorithm converges. For the time-invariant SISO system given by (1) and (11), this relation can using transfer operators be rewritten to

$$
e_{\infty}(t)=\left(1-T_{y z}^{0}(q) T_{y z}^{-1}(q)\right) r(t)
$$

From this expression it can be seen that when the nominal model $T_{y z}$ is equal to the true system $T_{y z}^{0}$, it gives $e_{\infty}=0$. An obvious question is how close $T_{y z}$ needs to be to $T_{y z}^{0}$ to get better performance using the approach in case $\mathrm{B}$ compared to case A. This will depend on the properties of each particular application.

\section{Case C - ILC using estimate from linear observer}

The estimate $\hat{z}_{k}$ is now given by (26),

$$
\hat{\boldsymbol{z}}_{k}=\boldsymbol{F}_{r} \boldsymbol{r}+\boldsymbol{F}_{u} \boldsymbol{u}_{k}+\boldsymbol{F}_{y} \boldsymbol{y}_{k}
$$

Using $Q=I$ and under the assumption that the ILC algorithm converges, the asymptotic error $\boldsymbol{e}_{\infty}$ is from Lemma 3 given by

$$
\begin{aligned}
\boldsymbol{e}_{\infty}=\left(I-\boldsymbol{T}_{r z}^{0}-\boldsymbol{T}_{u z}^{0}\left(\boldsymbol{L}\left(\boldsymbol{F}_{u}+\boldsymbol{F}_{y} \boldsymbol{T}_{u y}^{0}\right)\right)^{-1}\right. \\
\left.\cdot \boldsymbol{L}\left(I-\left(\boldsymbol{F}_{r}+\boldsymbol{F}_{y} \boldsymbol{T}_{r y}^{0}\right)\right)\right) \boldsymbol{r}
\end{aligned}
$$

Under the assumption that the relation (31) holds, it can for the time-invariant SISO system in (1) and (11) be rewritten in transfer-operator form as

$$
e_{\infty}=\left(1-T_{y z}^{0}(q) \frac{T_{r y}^{0}(q) F_{u}(q)+\left(1-F_{r}(q)\right) T_{u y}^{0}(q)}{F_{u}(q)+F_{y}(q) T_{u y}^{0}(q)}\right)
$$

where the argument $t$ has been temporarily omitted. Now use the relation (14) from the observer (12) in Example 5,

$$
\begin{aligned}
\hat{z}_{k}(t)= & F_{\bar{u}}(q) F(q) r(t)+F_{\bar{u}}(q) F(q) u_{k}(t) \\
& +\left(F_{\bar{y}}(q)-F_{\bar{u}}(q) F(q)\right) y_{k}(t)
\end{aligned}
$$

where $F_{\bar{u}}(q)$ and $F_{\bar{y}}(q)$ are given by (13). The closed-loop system is from Example 2 given by (5),

$$
y_{k}(t)=\underbrace{\frac{F(q) T_{\bar{u} y}^{0}(q)}{1+F(q) T_{\bar{u} y}^{0}(q)}}_{T_{r y}^{0}(q)} r(t)+\underbrace{\frac{F(q) T_{\bar{u} y}^{0}(q)}{1+F(q) T_{\bar{u} y}^{0}(q)}}_{T_{u y}^{0}(q)} u_{k}(t)
$$

where the transfer operators in (4) are denoted $T_{\bar{u} y}^{0}$ and $T_{\bar{u} z}^{0}$ for the system with input $\bar{u}_{k}$. The asymptotic error $e_{\infty}$ in (32) is after some manipulation then

$$
e_{\infty}(t)=\left(1-T_{y z}^{0}(q) \frac{T_{\bar{u} y}^{0}(q)}{F_{\bar{u}}(q)+F_{\bar{y}}(q) T_{\bar{u} y}^{0}(q)}\right) r(t)
$$

Assuming $\bar{u}_{k} \neq 0$, thereby implicitly $F \neq 0$, it can be noted that the expression for $e_{\infty}$ in (33) is independent of the controller $F$.

If the observer is based on the true system, the numerator of (33) is

$$
\begin{aligned}
F_{\bar{u}}(q)+F_{\bar{y}}(q) T_{\bar{u} y}^{0}(q)=M^{0}\left(q I-\left(A^{0}-K C^{0}\right)\right)^{-1} B^{0} \\
\quad+M^{0}\left(q I-\left(A^{0}-K C^{0}\right)\right)^{-1} K C^{0}\left(q I-A^{0}\right)^{-1} B^{0} \\
=M^{0}\left(q I-\left(A^{0}-K C^{0}\right)\right)^{-1}\left(q I-A^{0}+K C^{0}\right) \\
\quad \cdot\left(q I-A^{0}\right)^{-1} B^{0} \\
=M^{0}\left(q I-A^{0}\right)^{-1} B^{0}=T_{\bar{u} z}^{0}(q)=T_{y z}^{0}(q) T_{\bar{u} y}^{0}(q)
\end{aligned}
$$

where the last row can be realised from (11). From this expression it is seen that the asymptotic error $e_{\infty}=0$ in (33), which is expected when $\hat{z}_{k}$ results from an observer based on the true system 
A topic for future work is the discussion of how to choose the observer gain $K$ in case of model errors to achieve the best possible performance. Another interesting question is how case $\mathrm{C}$ holds compared to the approach in case $\mathrm{B}$ when having model errors.

\section{Case D-ILC using controlled variable}

Now the last case, which is used to show what ideally can be achieved compared to the cases $\mathrm{A}-\mathrm{C}$ discussed above. For this case the error $\boldsymbol{\epsilon}_{k}=\boldsymbol{r}-\boldsymbol{z}_{k}$ in (25) is used in the ILC algorithm (24). The asymptotic error $e_{\infty}$ of the controlled variable is from Lemma 3 given by

$$
\begin{array}{r}
\boldsymbol{e}_{\infty}=\left(I-\boldsymbol{T}_{r z}^{0}-\boldsymbol{T}_{u z}^{0}\left(I-\boldsymbol{Q}\left(I-\boldsymbol{L} \boldsymbol{T}_{u z}^{0}\right)\right)^{-1}\right. \\
\left.\cdot \boldsymbol{Q L}\left(I-\boldsymbol{T}_{r z}^{0}\right)\right) \boldsymbol{r}
\end{array}
$$

This expression can for a SISO time-invariant system described in transfer-operator form (1) - (2) be simplified to

$$
e_{\infty}(t)=\frac{(1-Q(q))\left(1-T_{r z}^{0}(q)\right)}{1-Q(q)\left(1-L(q) T_{u z}^{0}(q)\right)} r(t)
$$

Clearly this expression is zero when the ILC algorithm converges for the choice $Q=1$, achieving perfect tracking of the controlled variable $z_{k}$.

From Sections VI-B to VI-D it can be concluded that the asymptotic error $e_{\infty}=0$ when the estimate $\hat{z}_{k}$ is based on the true system, and using $Q=1$ in the ILC algorithm. However, using the approach in case A, Section VI-A, results in an asymptotic error $e_{\infty} \neq 0$ whenever $T_{y z}^{0} \neq 1$.

\section{CONCLUSIONS AND FUTURE WORK}

In this paper a framework for analysis of an ILC algorithm based on an estimate of the controlled variable obtained from an observer-based estimation procedure is proposed. The need of such a framework is motivated by the fact that in some applications the controlled variable is not the measured variable. The description is a natural extension of the system description presented in [11] and is focusing on the performance of the controlled variable. A general expression for the asymptotic error of the controlled variable when the ILC algorithm has converged is derived using the framework. The asymptotic error is discussed for three cases, especially if the error used in the ILC update goes to zero, and is exemplified by an ILC algorithm applied to a flexible two-mass model of a robot joint.

Further work includes analysis of performance when the ILC algorithm is based on an estimate of the controlled variable in presence of model errors, and also which error that is the best to use in the ILC algorithm from performance point of view when having model errors. Another issue to be discussed is how performance is affected by the choice of input point of the ILC algorithm to the system.

\section{APPENDIX}

The two-mass system, illustrated in Fig. 6, is a common first approximation of a mechanical system. The input $\bar{u}(t)$ is the torque applied to the first mass. The angular position $y(t)$ of the first mass, also referred to as motor angle, is the measured variable, and the angular position $z(t)$ of the second mass, called arm angle, is the controlled variable. The

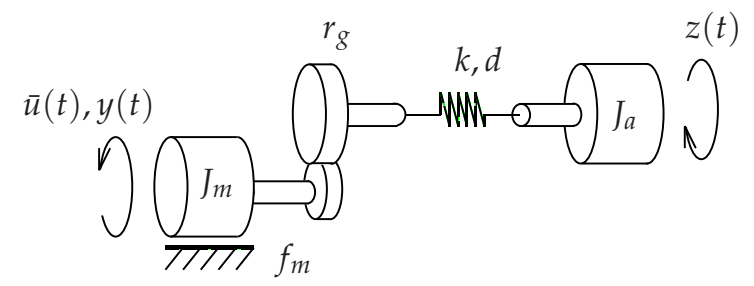

Fig. 6. A flexible two-mass model of a mechanical system, for example a single robot joint, characterised by spring $k$, damper $d$, viscous friction $f_{m}$, gear ratio $r_{g}$, moments of inertia $J_{m}, J_{a}$, torque $\bar{u}(t)$, motor angle $y(t)$ and arm angle $z(t)$.

two-mass system is described by the following equations

$$
\begin{aligned}
J_{m} \ddot{y}(t)= & -f_{m} \dot{y}(t)-r_{g} k\left(r_{g} y(t)-z(t)\right) \\
& -r_{g} d\left(r_{g} \dot{y}(t)-\dot{z}(t)\right)+k_{u} \bar{u}(t) \\
J_{a} \ddot{z}(t)= & k\left(r_{g} y(t)-z(t)\right)+d\left(r_{g} \dot{y}(t)-\dot{z}(t)\right)
\end{aligned}
$$

with spring coefficient $k$, damping coefficient $d$, viscous friction $f_{m}$, gear ratio $r_{g}$, and moments of inertia $J_{m}$ and $J_{a}$. A state-space description of the two-mass system can be derived using, for example, the states

$$
x(t)=\left(\begin{array}{llll}
z(t) & \dot{z}(t) & y(t) \quad \dot{y}(t))^{T}
\end{array}\right.
$$

From (34) it is straightforward to compute the following transfer functions

$$
\begin{aligned}
& y(t)=T_{\bar{u} y}^{0}(p) \bar{u}(t) \\
& z(t)=T_{y z}^{0}(p) y(t)
\end{aligned}
$$

where $p$ is the derivative operator. In Fig. 7 a block diagram with a system given by the structure (35) - (36) is illustrated. The system $T_{\bar{u} y}^{0}$ is stabilised by a controller $F$ and the ILC input $u_{k}$ at iteration $k$ is added to the reference $r$.

It is not possible to find an exact discrete-time representation of the relation between $y(t)$ and $z(t)$ in (36) since $y(t)$ is an internal continuous variable in the system described

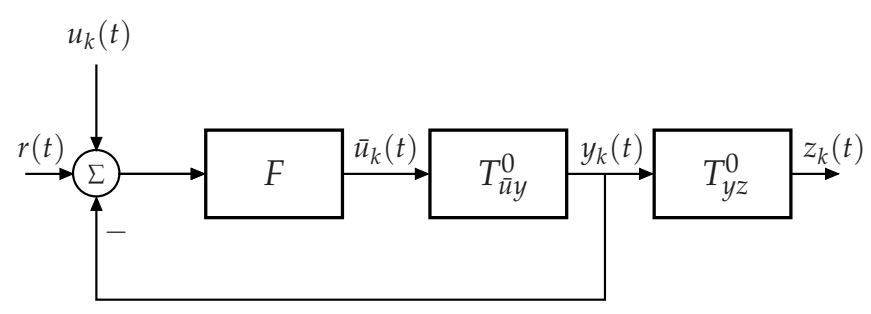

Fig. 7. The two-mass system illustrated by $T_{\bar{u} y}^{0}$ and $T_{y z}^{0}$; transfer function from motor torque $\bar{u}_{k}(t)$ to motor angle $y_{k}(t)$ and from motor angle $y_{k}(t)$ to arm angle $z_{k}(t)$, respectively, controlled by the feedback controller $F$. The ILC input $u_{k}(t)$ is added to the reference $r(t)$ at iteration $k$. 
by (34). When the sampling time is short it is however possible to find a good approximation, using for example the Tustin derivative approximation.

\section{REFERENCES}

[1] S. Arimoto, S. Kawamura, and F. Miyazaki. Bettering operation of robots by learning. Journal Robot. Syst., 1(2):123-140, 1984.

[2] G. Casalino and G. Bartolini. A learning procedure for the control of movements of robotic manipulators. In Proc. IASTED Sym. Robot. Autom., pages 108-111, San Francisco, USA, May 1984.

[3] J. J. Craig. Adaptive control of manipulators through repeated trials. In Proc. American Control Conf., pages 1566-1572, San Diego, CA, USA, June 1984.

[4] D. A. Bristow, M. Tharayil, and A. G. Alleyne. A survey of iterative learning control. IEEE Control Syst. Mag., pages 96-114, June 2006.

[5] J. Wallén, M. Norrlöf, and S. Gunnarsson. Arm-side evaluation of ILC applied to a six-degrees-of-freedom industrial robot. In Proc. IFAC World Congress, pages 13450-13455, Seoul, Korea, July 2008. Invited paper.

[6] ABB, 2008. URL: http: / /www . abb. se, accessed October, 2008

[7] S. Gunnarsson, M. Norrlöf, E. Rahic, and M. Özbek. On the use of accelerometers in iterative learning control of a flexible robot arm. Int. Journal Control, 80(3):363-373, March 2007.
[8] J. Wallén, S. Gunnarsson, R. Henriksson, S. Moberg, and M. Norrlöf ILC applied to a flexible two-link robot model using sensor-fusionbased estimates. Accepted for publication in Proc. IEEE Conf. Decision Control, Shanghai, China, December 2009.

[9] A. Schöllig and R. D’Andrea. Optimization-based iterative learning control for trajectory tracking. In Proc. Eur. Control Conf., pages 1505-1510, Budapest, Hungary, August 2009.

[10] A. Tayebi and J.-X. Xu. Observer-based iterative learning control for a class of time-varying nonlinear systems. IEEE Trans. Circuits Systems I: Fundamental Theory Appl., 50(3):452-455, March 2003.

[11] M. Norrlöf and S. Gunnarsson. Time and frequency domain convergence properties in iterative learning control. Int. Journal Control, 75:1114-1126, 2002.

[12] M. Phan and R. W. Longman. A mathematical theory of learning control of linear discrete multivariable systems. In Proc. AIAA/AAS Astrodyn. Conf., pages 740-746, Minneapolis, MN, USA, August 1988

[13] Kevin L. Moore. Multi-loop control approach to designing iterative learning controllers. In Proc. IEEE Conf. Decision Control, pages 151-214, Tampa, FL, USA, December 1998.

[14] R. Tousain, E. van der Meché, and O. Bosgra. Design strategy for iterative learning control based on optimal control. In Proc. IEEE Conf. Decision Control, Orlando, FL, USA, December 2001.

[15] T. Kailath, A. H. Sayed, and B. Hassibi. Linear estimation. PrenticeHall, Inc, Upper Saddle River, NJ, USA, 2000. 


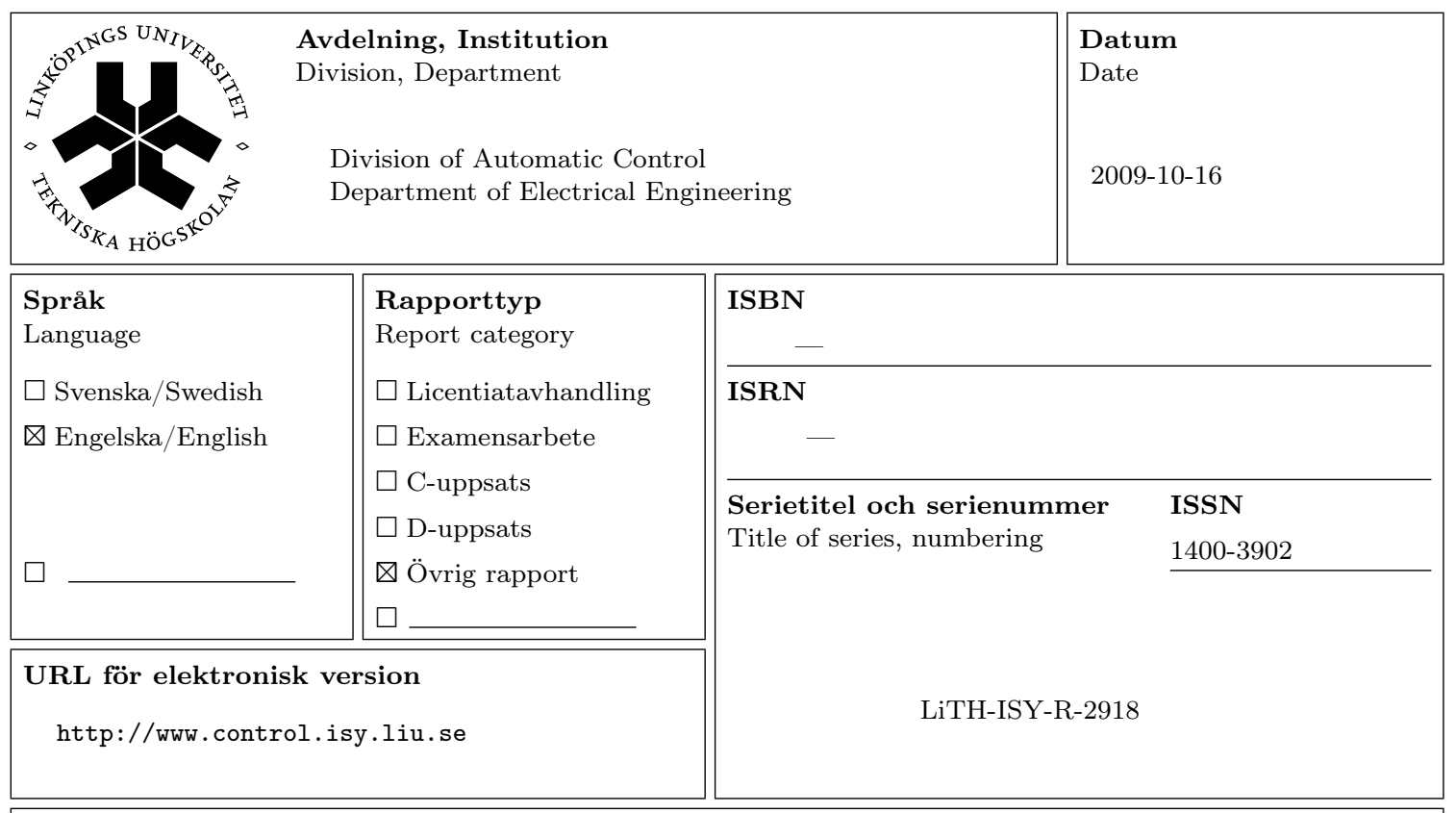

Titel A framework for analysis of observer-based ILC

Title

A framework for analysis of observer-based ILC

Författare Johanna Wallén, Mikael Norrlöf, Svante Gunnarsson

Author

\section{Sammanfattning}

Abstract

A framework for Iterative Learning Control (ILC) is proposed for the situation when the ILC algorithm is based on an estimate of the controlled variable obtained from an observer-based estimation procedure. Under the assumption that the ILC input converges to a bounded signal, a general expression for the asymptotic error of the controlled variable is given. The asymptotic error is then exemplified by an ILC algorithm applied to a flexible two-mass model of a robot joint. 